\title{
Linearization of multi-objective multi-quadratic 0-1 programming problems
}

\author{
Shifali Bhargava \\ Department of Mathematics, B.S.A. College, Mathura (U.P)-India \\ E-mail: shifalibhargava@gmail.com
}

Copyright $\odot 2014$ Shifali Bhargava. This is an open access article distributed under the Creative Commons Attribution License, which permits unrestricted use, distribution, and reproduction in any medium, provided the original work is properly cited.

\begin{abstract}
A linearization technique is developed for multi-objective multi-quadratic 0-1 programming problems with linear and quadratic constraints to reduce it to multi-objective linear mixed 0-1 programming problems. The method proposed in this paper needs only $\mathrm{O}(\mathrm{kn})$ additional continuous variables where $\mathrm{k}$ is the number of quadratic constraints and $\mathrm{n}$ is the number of initial 0-1 variables.
\end{abstract}

Keywords: Knapsack Constraint, Linearization, Multi-Objective, Multi-Quadratic, Optimal Solution.

\section{Introduction}

Multi-objective Multi-quadratic Integer Programming (MOMQIP) plays an important modeling role for many diverse problems. This is a structured global optimization problem, which encompasses many others. Many optimization problems can easily be reformulated as special cases of MOMQIP. However, there are theoretical and practical difficulties in the process of solving such problems. Finding an exact and finite algorithm that solves large MOMQIP problems is hard. However, the MOMQIP constitutes an important part of mathematical programming problems, arising in various practical applications including facility location, production planning, VLSI chip design, optimal design of water distribution networks and medical applications. [6], [7], [9].

Here in this paper, the multi-objective multi-quadratic programming problem with quadratic and linear constraints is considered. There are many different techniques to solve general quadratic integer programming problems. Most of them are of branch and bound type or some type of linearization techniques. A lot of linearization techniques have been discussed in literature. [1] Some of the techniques are focused on providing concise models and tightening constraint bounds whereas some linearization techniques are based on the restriction of positive denominators. These requires less number of constraints, variables and auxiliary constraints as compared to the available techniques in the literature.[2], [10], [13] A linearization technique in which the auxiliary constraints involving binary variables are used in some cases of the transformed models to restrict the repetition of goals has been proposed. [11] A disadvantage of the standard technique, where we introduce an additional variable for each product $\mathrm{x}_{\mathrm{i}} \mathrm{x}_{\mathrm{j}}$ is that number of new variables is $\mathrm{O}\left(\mathrm{n}^{2}\right)$ where $n$ is the number of initial 0-1 variables.[3], [4], [5], [12] A linearization technique for multi-quadratic 0-1 programming problem has been proposed earlier [8]. Here this work is extended for multi-objective multi-quadratic programming problems. The method proposed in this paper needs only $\mathrm{O}(\mathrm{kn})$ additional continuous variables, where $\mathrm{k}$ is the number of quadratic constraints and the number of initial 0-1 variables remains the same. For $\mathrm{k}=\mathrm{o}(\mathrm{n})$ the linearization techniques proposed in the paper introduces less number of additional variables. The number of additional linear constraints is $\mathrm{O}(\mathrm{kn})$. This technique can be applied to obtain new linear $0-1$ formulations for combinatorial optimization problems which can be formulated as quadratic 0-1 programming problems.

\section{Main results}

The multi- objective multi-quadratic 0-1 programming problem can be rewritten as: 
$\min _{x \in\{0,1\}^{n}} f_{1}(x)=x^{T} A_{1} x$
$\min _{x \in\{0,1\}^{n}} f_{2}(x)=x^{T} A_{2} x$
$\vdots$
$\min _{x \in\{0,1\}^{n}} f_{r}(x)=x^{T} A_{r} x$

s.t. $\quad B x \geq b$,

$\mathrm{x}^{\mathrm{T}} \mathrm{C}_{1} \mathrm{x} \geq \alpha_{1}$

$\mathrm{x}^{\mathrm{T}} \mathrm{C}_{2} \mathrm{x} \geq \alpha_{2}$

$\mathrm{x}^{\mathrm{T}} \mathrm{C}_{3} \mathrm{x} \geq \alpha_{3}$

$\mathrm{x}^{\mathrm{T}} \mathrm{C}_{\mathrm{k}} \mathrm{x} \geq \alpha_{\mathrm{k}}$

Where $A_{j} \in R^{n \times n}, \quad C_{i} \in R^{n x n}, \alpha_{i} \in R, \quad \forall j=1,2, \ldots r$ and $\forall i=1,2, \ldots k$. Here $k$ is the number of quadratic constraints, where $\mathrm{k}$ is some non-negative integer number. $\mathrm{x} \in\{0,1\}^{\mathrm{n}}, \mathrm{B}$ is a matrix of linear constraints, $\mathrm{b}$ is a constant vector, $\mathrm{m}$ and $\mathrm{n}$ are some integers.

The paper formulation is as follows: First we examine the special case of the problem where all the matrices $A_{j}$, $\mathrm{j}=1,2, \ldots \mathrm{r}$ and all matrices of quadratic constraints are non-negative. In the next section, it is proved that on having a knapsack constraint, the general case can be reduced to the initial one i.e. when $A_{j}$ and $C_{i} \forall j=1,2, \ldots r$ and $\forall i=$ $1,2, \ldots \mathrm{k}$ are non-negative. Section 4 presents a linearization technique for the case of general matrices. At last, conclusions are drawn.

\section{Special Case}

Let us consider the multi-objective quadratic $0-1$ programming problem which has the form

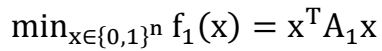

$\min _{\mathrm{x} \in\{0,1\}^{\mathrm{n}}} \mathrm{f}_{2}(\mathrm{x})=\mathrm{x}^{\mathrm{T}} \mathrm{A}_{2} \mathrm{x}$

$$
\vdots
$$

$\min _{x \in\{0,1\}^{n}} f_{r}(x)=x^{T} A_{r} x$

s.t.Bx $\geq \mathrm{b}$,

Where $A_{j} \in R^{n \times n}$ and each element of these matrices is non-negative. $x \in\{0,1\}^{n}, B$ is a matrix of linear constraints, $b$ is a constant vector, $\mathrm{m}$ and $\mathrm{n}$ are some integers. Let $\mathrm{e}$ be a vector of all1's, i.e. $\mathrm{e}=(1,1, \ldots, 1)^{\mathrm{T}}$.

Consider the following two problems $\mathrm{P}_{1}$ and $\overline{\mathrm{P}_{1}}$ with linear constraints and prove that these are equivalent.

Problem $P_{1}$

$$
\begin{aligned}
& \min _{x} f_{1}(x)=x^{T} A_{1} x \\
& \min _{x} f_{2}(x)=x^{T} A_{2} x \\
& \vdots \\
& \min _{\mathrm{x}} \mathrm{f}_{\mathrm{r}}(\mathrm{x})=\mathrm{x}^{\mathrm{T}} \mathrm{A}_{\mathrm{r}} \mathrm{x} \\
& \text { s.t. } B x \geq b, x \in\{0,1\}^{n} \\
& \text { Problem } \bar{P}_{1} \\
& \min _{\mathrm{x}, \mathrm{y}_{1}, \mathrm{~s}_{1}} \mathrm{~g}_{1}(\mathrm{~s})=\mathrm{e}^{\mathrm{T}} \mathrm{s}_{1} \\
& \min _{x, y_{2}, s_{2}} g_{2}(s)=e^{T} s_{2} \\
& \mathrm{x}, \mathrm{y}_{2,} \mathrm{~s}_{2}
\end{aligned}
$$

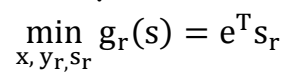

$$
\begin{aligned}
& \text { s.t. } \\
& \mathrm{A}_{1} \mathrm{x}-\mathrm{y}_{1}-\mathrm{s}_{1}=0 \\
& \mathrm{~A}_{2} \mathrm{x}-\mathrm{y}_{2}-\mathrm{s}_{2}=0 \\
& \mathrm{~A}_{\mathrm{r}} \mathrm{x}-\mathrm{y}_{\mathrm{r}}-\mathrm{s}_{\mathrm{r}}=0 \\
& B x \geq b, \quad y_{j}^{T} x=0, \forall j=1,2, \ldots r, x \in\{0,1\}^{n}, y_{j p} \geq 0, s_{j p} \geq 0
\end{aligned}
$$

Theorem 2.1: $\mathrm{P}_{1}$ has a Pareto optimal solution $\mathbf{x}^{\mathbf{0}}$ iff there exist $\mathbf{y}^{\mathbf{0}}, \mathbf{s}^{\mathbf{0}}$ such that $\left(\mathbf{x}^{\mathbf{0}}, \mathbf{y}^{\mathbf{0}}, \mathbf{s}^{\mathbf{0}}\right)$ is a pareto optimal solution of $\overline{\mathrm{P}_{1}}$.

Proof: Let $\mathbf{x}^{\mathbf{0}}$ be a Pareto optimal solution of the problemP $P_{1}$. Note that since all elements of the matrices $\mathbf{A}_{\mathbf{p}}$ are nonnegative, it is obvious that there exist $y, s$ s.t $y_{p} \geq 0, s_{p} \geq 0$ and

$A_{1} x-y_{1}-s_{1}=0, \quad A_{2} x-y_{2}-s_{2}=0, \ldots, \quad A_{r} x-y_{r}-s_{r}=0$

$\mathrm{y}^{\mathrm{T}} \mathrm{x}^{0}=0$

Choose $\mathrm{y}^{0}, \mathrm{~s}^{0}$ from the above defined set of $\mathrm{y}$ and $\mathrm{s}$ such that the value of $\mathrm{e}^{\mathrm{T}} \mathrm{s}^{0}$ is minimized. It remains to prove that $\left(\mathrm{x}^{0}, \mathrm{y}^{0}, \mathrm{~s}^{0}\right)$ is an optimal solution of the problem $\overline{\mathrm{P}_{1}}$.

Multiplying (2) by $\left(x^{0}\right)^{\mathrm{T}}$, we obtain

$\left(x^{0}\right)^{\mathrm{T}} \mathrm{A}_{1} \mathrm{x}^{0}-\left(\mathrm{x}^{0}\right)^{\mathrm{T}} \mathrm{y}_{1}{ }^{0}-\left(\mathrm{x}^{0}\right)^{\mathrm{T}} \mathrm{s}_{1}{ }^{0}=0$ 
$\left(\mathrm{x}^{0}\right)^{\mathrm{T}} \mathrm{A}_{2} \mathrm{x}^{0}-\left(\mathrm{x}^{0}\right)^{\mathrm{T}} \mathrm{y}_{2}{ }^{0}-\left(\mathrm{x}^{0}\right)^{\mathrm{T}} \mathrm{s}_{2}{ }^{0}=0$,

$\left(x^{0}\right)^{\mathrm{T}} A_{r} x^{0}-\left(x^{0}\right)^{\mathrm{T}} y_{r}{ }^{0}-\left(x^{0}\right)^{\mathrm{T}} s_{r}{ }^{0}=0$

By using (3),

$\left(\mathrm{x}^{0}\right)^{\mathrm{T}} \mathrm{A}_{1} \mathrm{x}^{0}=\left(\mathrm{x}^{0}\right)^{\mathrm{T}} \mathrm{s}_{1}{ }^{0}$,

$\left(x^{0}\right)^{\mathrm{T}} A_{2} x^{0}=\left(x^{0}\right)^{\mathrm{T}} s_{2}{ }^{0}$,

$\left(\mathrm{x}^{0}\right)^{\mathrm{T}} \mathrm{A}_{\mathrm{r}} \mathrm{x}^{0}=\left(\mathrm{x}^{0}\right)^{\mathrm{T}} \mathrm{S}_{\mathrm{r}}{ }^{0}$

Now $\left(\mathrm{x}^{0}, \mathrm{y}^{0}, \mathrm{~s}^{0}\right)$ will be an optimal solution of $\overline{\mathrm{P}_{1}}$ if

$\left(\mathrm{x}^{0}\right)^{\mathrm{T}} \mathrm{s}_{\mathrm{p}}{ }^{0}=\mathrm{e}^{\mathrm{T}} \mathrm{s}_{\mathrm{p}}{ }^{0}$

To prove that (6) holds, it is sufficient to show that, for any $\mathrm{p}$ if $\mathrm{x}_{\mathrm{p}}=0$ then $\mathrm{s}_{\mathrm{p}}=0$. It is proved now by contradiction. Assume now that for some $p$, we have that $x_{p}{ }^{0}=0 \& s_{p}{ }^{0} \geq 0$ where $\left(y^{0}, s^{0}\right)$ were chosen to minimize $e^{T} s_{p}{ }^{0}$. Let us define vector $\bar{y}$ and $\bar{s}$ as $\overline{y_{p}}=y_{p}^{0}+s_{p}^{0}, \overline{s_{p}}=0$ and for $\mathrm{p} \neq \mathrm{q}, \overline{y_{q}}=y_{q}$ and $\overline{s_{q}}=s_{q}$.

It is easy to check that $\left(\mathrm{x}_{\mathrm{p}}^{0}, \overline{\mathrm{y}_{\mathrm{q}}}, \overline{\mathrm{s}_{\mathrm{q}}}\right)$ also satisfies (2), (3) and $\mathrm{e}^{\mathrm{T}} \overline{\mathrm{s}_{\mathrm{p}}}<e^{\mathrm{T}} \mathrm{s}_{\mathrm{p}}{ }^{0}$. this contradicts with the initial assumption that $\mathrm{y}_{\mathrm{p}}^{0}$ and $\mathrm{s}_{\mathrm{p}}^{0}$ were choosen to minimize $\mathrm{e}^{\mathrm{T}} \mathrm{s}_{\mathrm{p}}{ }^{0}$. Similarly the converse can be proved.

Reformulation of Problem $\overline{\mathrm{P}_{1}}$

It is easy to see from the complementarity constraint $y^{T} x=0$ that $\forall$ p where $x_{p}=1$, we need to have $y_{p}=0$; for every $p$, where $x_{p}=0$, the value of $y_{p}$ does not depend on this constraint. Also note from (2) that the value of $y_{p}$ is bounded above by the value of

$\mathrm{M}_{1}=\min _{\mathrm{i}} \sum_{\mathrm{j}=1}^{\mathrm{n}}\left|\mathrm{a}_{1 \mathrm{ij}}\right|=\left\|\mathrm{A}_{1}\right\|_{\infty}$

$\mathrm{M}_{2}=\min _{\mathrm{i}} \sum_{\mathrm{j}=1}^{\mathrm{n}}\left|\mathrm{a}_{2 \mathrm{ij}}\right|=\left\|\mathrm{A}_{2}\right\|_{\infty}$

$\mathrm{M}_{\mathrm{r}}=\min _{\mathrm{i}} \sum_{\mathrm{j}=1}^{\mathrm{n}}\left|\mathrm{a}_{\mathrm{rij}}\right|=\left\|\mathrm{A}_{\mathrm{r}}\right\|_{\infty}$

Therefore, $\overline{\mathrm{P}}_{1}$ can be reformulated as a linear mixed 0-1 programming problem by replacing $\mathrm{y}^{\mathrm{T}} \mathrm{x}^{0}=0$ by linear constraints $\mathrm{y}_{1} \leq \mathrm{M}_{1}(\mathrm{e}-\mathrm{x}), \mathrm{y}_{2} \leq \mathrm{M}_{2}(\mathrm{e}-\mathrm{x}), \ldots, \mathrm{y}_{\mathrm{r}} \leq \mathrm{M}_{\mathrm{r}}(\mathrm{e}-\mathrm{x})$.

As a result, the following formulation is obtained:

$\min g(s)=\mathrm{e}^{\mathrm{T}} \mathrm{s}_{\mathrm{u}}$

s.t.

$\mathrm{A}_{1} \mathrm{x}-\mathrm{y}_{1}-\mathrm{s}_{1}=0$

$\mathrm{A}_{2} \mathrm{x}-\mathrm{y}_{2}-\mathrm{s}_{2}=0$,

$A_{\mathrm{r}} \mathrm{x}-\mathrm{y}_{\mathrm{r}}-\mathrm{s}_{\mathrm{r}}=0$

$\mathrm{Bx} \geq \mathrm{b}$

$\mathrm{y}_{1} \leq \mathrm{M}_{1}(\mathrm{e}-\mathrm{x}), \mathrm{y}_{2} \leq \mathrm{M}_{2}(\mathrm{e}-\mathrm{x}), \ldots, \mathrm{y}_{\mathrm{r}} \leq \mathrm{M}_{\mathrm{r}}(\mathrm{e}-\mathrm{x})$

$\mathrm{x} \in\{0,1\}^{\mathrm{n}}, \mathrm{y}_{\mathrm{u}} \geq 0, \mathrm{~s}_{\mathrm{u}} \geq 0$

Now we discuss multi- objective multi-quadratic $0-1$ programming problem with quadratic constraints. Let $C_{i} \in R^{\text {nxn }}$ whose each element of every matrix is positive.

Consider the following two problems $\mathrm{P}_{2}$ and $\overline{\mathrm{P}_{2}}$ :

Problem $\mathrm{P}_{2}$

$\min _{x \in\{0,1\}^{n}} f_{1}(x)=x^{\mathrm{T}} A_{1} x$
$\min _{x \in\{0,1\}^{n}} f_{2}(x)=x^{\mathrm{T}} A_{2} x$
$\vdots$
$\min _{x \in\{0,1\}^{n}} f_{r}(x)=x^{\mathrm{T}} A_{r} x$

S.t. $B x \geq b, x^{T} C_{1} x \geq \alpha_{1}, x^{T} C_{2} x \geq \alpha_{2}, \ldots, x^{T} C_{k} x \geq \alpha_{k}, x \in\{0,1\}^{n}, \alpha_{i}$ is positive constants.

Problem $\overline{\mathrm{P}}_{2}$

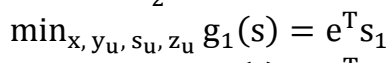

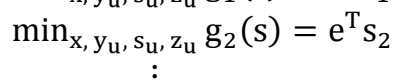

$\min _{x, y_{u}, s_{u}, z_{u}} g_{r}(s)=e^{T} s_{r}$

s.t. $A_{1} x-y_{1}-s_{1}=0$

$A_{2} x-y_{2}-s_{2}=0$

$A_{r} x-y_{r}-s_{r}=0$

$B x \geq b$,

$y_{1} \leq M_{1}(e-x), y_{2} \leq M_{2}(e-x), \ldots, y_{r} \leq M_{r}(e-x)$

$C_{1} x-z_{1} \geq 0, C_{2} x-z_{2} \geq 0, \ldots, C_{r} x-z_{r} \geq 0$

$\mathrm{e}^{T} z_{1} \geq \alpha_{1}, e^{T} z_{2} \geq \alpha_{2}, \ldots, e^{T} z_{k} \geq \alpha_{k}$

$z_{1} \leq M_{1}^{\prime} x, z_{2} \leq M_{2}^{\prime} x, \ldots, z_{k} \leq M_{k}^{\prime} x$

$x \in\{0,1\}^{n}, y_{u}, s_{u}, z_{i} \geq 0$ Where

$M_{1}^{\prime}=\left\|C_{1}\right\|_{\infty}, M_{2}^{\prime}=\left\|C_{2}\right\|_{\infty}, \ldots, M_{k}^{\prime}=\left\|C_{k}\right\|_{\infty}$, and

$M_{1}=\left\|A_{1}\right\|_{\infty}, M_{2}=\left\|A_{2}\right\|_{\infty}, \ldots, M_{r}=\left\|A_{r}\right\|_{\infty}$ 
Theorem 2.2: $P_{2}$ has a Pareto optimal solution $x^{0}$ iff there exist $y^{0}, s^{0}$ such that $\left(x^{0}, y_{u}{ }^{0}, s_{u}{ }^{0}, z_{i}{ }^{0}\right)$ is a pareto optimal solution of $\overline{P_{2}}$.

Proof: From the proof of Theorem 2.1, in order to extend the proof for the multi-quadratic case it is required to show that if $x^{0}$ is a Pareto optimal solution of the problem $P_{2}$ then there exists vectors $z_{i}{ }^{0}$ such that every component of every vector is non-negative and the following constraints are satisfied:

$C_{1} x^{0}-z_{1} \geq 0, C_{2} x^{0}-z_{2} \geq 0, \ldots, \mathrm{C}_{r} x^{0}-z_{r} \geq 0$

$e^{T} z_{1}{ }^{0} \geq \alpha_{1}, e^{T} z_{2}{ }^{0} \geq \alpha_{2}, \ldots, e^{T} z_{k}{ }^{0} \geq \alpha_{k}$

$z_{1}{ }^{0} \leq M_{1}^{\prime} x^{0}, z_{2}{ }^{0} \leq M_{2}^{\prime} x^{0}, \ldots, z_{k}{ }^{0} \leq M_{k}^{\prime} x^{0}$

By using (9), $x_{i}^{0}=0 \Rightarrow z_{i}^{0}=0$.

Similar to the proof of the Theorem 1, we have that

$e^{T} z_{1}{ }^{0} \geq\left(x^{0}\right)^{T} z_{1}{ }^{0}, e^{T} z_{2}{ }^{0} \geq\left(x^{0}\right)^{T} z_{2}{ }^{0}, \ldots, e^{T} z_{k}{ }^{0} \geq\left(x^{0}\right)^{T} z_{k}{ }^{0}$

Since $z_{i}{ }^{0}$ is a real no. and every element of the matrices $C_{i}$ is non-negative, then $\forall i$, where $x_{i}^{0}=1, z_{i}^{0} \geq 0$ can be chosen such that $C x_{i}^{0}=z_{i}^{0}$. Therefore (7) and (9) are satisfied.

Multiplying (7) by $\left(x^{0}\right)^{T}$, and from (10)

$e^{T} z_{1}{ }^{0}=\left(x^{0}\right)^{T} z_{1}{ }^{0}=\left(x^{0}\right)^{T} C_{1} x^{0}$

$e^{T} z_{2}{ }^{0}=\left(x^{0}\right)^{T} z_{2}{ }^{0}=\left(x^{0}\right)^{T} C_{2} x^{0}$

$e^{T} z_{k}{ }^{0}=\left(x^{0}\right)^{T} z_{k}{ }^{0}=\left(x^{0}\right)^{T} C_{k} x^{0}$

And as $x^{0}$ is a Pareto optimal solution of the problem $P_{2}$ then (8) is satisfied:

$\left(x^{0}\right)^{T} C_{1} x^{0}=e^{T} z_{1}{ }^{0} \geq \alpha_{1}$

$\left(x^{0}\right)^{T} C_{2} x^{0}=e^{T} z_{2}{ }^{0} \geq \alpha_{2}$

$\left(x^{0}\right)^{T} C_{k} x^{0}=e^{T} z_{k}{ }^{0} \geq \alpha_{k}$

\section{Knapsack constraint and reduction to the linear case}

Now the general case is considered when the elements of $A_{1}, A_{2}, \ldots, A_{r}$ and $C_{1}, C_{2}, \ldots, C_{k}$ can be negative. On having a knapsack constraint $w^{T} x=k$, where $\mathrm{k}$ are some constants and $w_{i}>1$, for $i=2, \ldots, n$, the problem can still be reduced to the equivalent one with matrices $A_{u}$ and $C_{u}$, whose every element of each matrix is positive i.e. the technique described in the previous section can be applied to linearize the problem. Let us show this reduction.

Without any loss of generality, let us assume that $w_{i} \geq 1$ for $\mathrm{i}=2, \ldots, n$.

Let $Q_{u} \in R^{n x n}$ and $Q_{u}=\left(\left(w w^{T}\right)_{u}\right)$. It is clear that every element of each matrix will be greater than equal to one. Also we define $\overline{\overline{A_{1}}}, \overline{\overline{A_{2}}}, \ldots \overline{\overline{A_{r}}}$ as follows.

$\overline{\overline{A_{1}}}=A_{1}+\max _{i, j}\left|a_{1 i j}\right| \cdot Q_{1}$

$\overline{\overline{A_{2}}}=A_{2}+\max _{i, j}\left|a_{2 i j}\right| \cdot Q_{2}$

$\vdots$

$\overline{\overline{A_{r}}}=A_{r}+\max _{i, j}\left|a_{r i j}\right| \cdot Q_{r}$

Since $q_{1 i j}, q_{2 i j}, \ldots, q_{r i j} \geq 1$, we have

$\overline{\overline{a_{1 \imath j}}}=a_{1 i j}+\max _{i, j}\left|a_{1 i j}\right| \cdot q_{1 i j}$

$\overline{\overline{a_{2 \imath j}}}=a_{2 i j}+\max _{i, j}\left|a_{2 i j}\right| \cdot q_{2 i j}$

$\vdots$

$\overline{\overline{a_{r l j}}}=a_{r i j}+\max _{i, j}\left|a_{r i j}\right| \cdot q_{r i j}$

Then

$x^{T} A_{1} x=x^{T}\left(\overline{\overline{A_{1}}}-\max _{i, j}\left|a_{1 i j}\right| \cdot Q_{1}\right) x=x^{T} A_{1} x-k^{2} \max _{i, j}\left|a_{1} i j\right| x^{T} A_{2} x=x^{T} A_{2} x-k^{2} \max _{i, j}\left|a_{2} i j\right|$

$x^{T} A_{r} x=x^{T} A_{r} x-k^{2} \max _{i, j}\left|a_{r} i j\right|$

Since the terms $k^{2} \max _{i, j}\left|a_{1} i j\right|, k^{2} \max _{i, j}\left|a_{2} i j\right|, \ldots, k^{2} \max _{i, j}\left|a_{r} i j\right|$ are constant, we can solve the initial problems using the matrices $\overline{\overline{A_{1}}}, \overline{\overline{A_{2}}}, \ldots, \overline{\overline{A_{r}}}$. The similar idea can be used to transform the quadratic constraints. Define $\overline{\overline{C_{1}}}$ and $\overline{\overline{\alpha_{1}}}$ as

$\overline{\overline{C_{1}}}=C_{1}+\max _{i, j}\left|c_{1} i j\right| \cdot Q_{1}$

$\overline{\overline{C_{2}}}=C_{2}+\max _{i, j}\left|c_{2} i j\right| \cdot Q_{1}$

:

$\overline{\overline{C_{k}}}=C_{k}+\max _{i, j}\left|c_{k} i j\right| . Q_{1}$

And

$\overline{\overline{\alpha_{1}}}=\alpha_{1}+k^{2} \max _{i, j}\left|c_{1} i j\right|$

$\overline{\overline{\alpha_{2}}}=\alpha_{2}+k^{2} \max _{i, j}\left|c_{2} i j\right|$ 
$\overline{\overline{\alpha_{k}}}=\alpha_{k}+k^{2} \max _{i, j}\left|c_{k} i j\right|$

It is clear that for every element of each matrix, $\overline{\overline{C_{1}}}$ is positive and the quadratic constraints can be reformulated as an equivalent one in the form

$x^{T} \overline{\overline{C_{1}}} x \geq \overline{\overline{\alpha_{1}}}, \quad x^{T} \overline{\overline{C_{2}}} x \geq \overline{\overline{\alpha_{2}}}, \quad \ldots \quad x^{T} \overline{\overline{C_{k}}} x \geq \overline{\overline{\alpha_{k}}}$

\section{General case}

In this section, a linearization technique is presented for the case of general matrices. Consider the formulation without quadratic constraints:

Problem $P_{3}$

$\min _{x \in\{0,1\}^{n}} f_{1}(x)=x^{T} A_{1} x$
$\min _{x \in\{0,1\}^{n}} f_{2}(x)=x^{T} A_{2} x$
$\vdots$
$\min _{x \in\{0,1\}^{n}} f_{r}(x)=x^{T} A_{r} x$

s.t. $B x \geq b, x \in\{0,1\}^{n}$

Problem $\overline{P_{3}}$

$\min _{x, y_{u}, s_{u}}, g_{1}(s)=e^{T} s_{1}-M_{1} e^{T} x$

$\min _{x, y_{u}, s_{u}}, g_{2}(s)=e^{T} s_{2}-M_{2} e^{T} x$

$$
\text { : }
$$

$\min _{x, y_{u}, s_{u}}, g_{r}(s)=e^{T} s_{r}-M_{r} e^{T} x$

s.t.

$A_{1} x^{0}-y_{1}-s_{1}+M_{1} e=0, A_{2} x-y_{2}-s_{2}+M_{2} e=0, \ldots, A_{r} x-y_{r}-s_{r}+M_{r} e=0, B x \geq b, x \in\{0,1\}^{n}$,

$y_{1} \leq 2 M_{1}(e-x), \quad y_{2} \leq 2 M_{2}(e-x), \ldots, \quad y_{r} \leq 2 M_{r}(e-x)$

$M_{1}=\max _{i} \sum_{j=1}^{n}\left|a_{1 i j}\right|=\left\|A_{1}\right\|_{\infty}$

$M_{2}=\max _{i} \sum_{j=1}^{n}\left|a_{2 i j}\right|=\left\|A_{2}\right\|_{\infty}$

$M_{r}=\max _{i} \sum_{j=1}^{n}\left|a_{r i j}\right|=\left\|A_{r}\right\|_{\infty} y_{u}, s_{u} \geq 0$

Theorem 4.1: $P_{3}$ has a Pareto optimal solution $x^{0}$ iff there existed $y^{0}, s^{0}$ such that $\left(x^{0}, y_{u}{ }^{0}, s_{u}{ }^{0}\right)$ is a pareto optimal solution of $\overline{P_{3}}$.

Proof: Necessity. Let $x^{0}$ be a Pareto optimal solution of the problem $P_{3}$. We need to prove that $\exists y_{u}, s_{u} \geq 0$ such that

$A_{i} x^{0}-y_{i}-s_{i}+M_{i} e=0, \forall i=1,2 \ldots r$

$y_{1}^{T} x^{0}=0, y_{2}^{T} x^{0}=0, \ldots, y_{r}^{T} \mathrm{x}^{0}=0$

As $M_{1}=\max _{1} \sum_{j=1}^{n}\left|a_{1} i j\right|$ then $A_{1} x^{0}+M_{1} e \geq 0$. Similarly this can be written for $\mathrm{M}_{2} \ldots \mathrm{M}_{\mathrm{r}}$. It is always possible to find $\mathrm{y}, \mathrm{s}$ such that $y_{u}, s_{u} \geq 0$ such that the needed equations (13) and (14) hold. Choose $y_{u}{ }^{0}, s_{u}{ }^{0}$ from the above defined set of $y_{u}, s_{u}$ such that $e^{T} s_{u}{ }^{0}$ is minimized. Then we prove that $\left(x^{0}, y_{u}{ }^{0}, s_{u}{ }^{0}\right)$ is a Pareto optimal solution of the problem $\overline{P_{3}}$. On multiplying (13) by $\left(x^{0}\right)^{T}$,

$\left(x^{0}\right)^{T} A_{1} x^{0}-\left(x^{0}\right)^{T} y_{1}-\left(x^{0}\right)^{T} s_{1}+M_{1}\left(x^{0}\right)^{T} e=0$

$\left(x^{0}\right)^{T} A_{2} x^{0}-\left(x^{0}\right)^{T} y_{2}-\left(x^{0}\right)^{T} s_{2}+M_{2}\left(x^{0}\right)^{T} e=0$

$\left(x^{0}\right)^{T} A_{r} x^{0}-\left(x^{0}\right)^{T} y_{r}-\left(x^{0}\right)^{T} s_{r}+M_{r}\left(x^{0}\right)^{T} e=0$

Note from (14) that $\left(x^{0}\right)^{T} y_{1}^{0}=0,\left(x^{0}\right)^{T} y_{2}^{0}=0, \ldots,\left(x^{0}\right)^{T} y_{r}^{0}=0$

Hence

$\left(x^{0}\right)^{T} A_{1} x^{0}=\left(x^{0}\right)^{T} s_{1}-M_{1}\left(x^{0}\right)^{T} e$

$\left(x^{0}\right)^{T} A_{2} x^{0}=\left(x^{0}\right)^{T} s_{2}-M_{2}\left(x^{0}\right)^{T} e$

$\left(x^{0}\right)^{T} A_{r} x^{0}=\left(x^{0}\right)^{T} s_{r}-M_{r}\left(x^{0}\right)^{T} e$

The rest of the proof is similar to the proof of the Theorem 2.1. Applying the same method (by contradiction) we can prove that

$e^{T}{S_{1}}^{0}=e\left({s_{1}}^{0}\right)^{T}, e^{T}{S_{2}}^{0}=e\left({s_{2}}^{0}\right)^{T}, \ldots, e^{T}{S_{r}}^{0}=e\left({s_{r}}^{0}\right)^{T}$

Hence using the fact that $e^{T} x^{0}=e\left(x^{0}\right)^{T}$, we obtain

$(e)^{T} A_{1} x^{0}=(e)^{T} s_{1}{ }^{0}-M_{1}(e)^{T} x^{0}$

$(e)^{T} A_{2} x^{0}=(e)^{T} s_{2}{ }^{0}-M_{2}(e)^{T} x^{0}$

$(e)^{T} A_{r} x^{0}=(e)^{T} s_{r}{ }^{0}-M_{r}(e)^{T} x^{0}$

Sufficiency: The proof is similar. In this case, on considering quadratic constraints $x^{T} C_{1} x \geq \alpha_{1}, x^{T} C_{2} x \geq \alpha_{2}, \ldots$, $x^{T} C_{k} x \geq \alpha_{k}$ the similar idea can be used. The following formulation is obtained:

Problem $P_{4}$

$\min _{x \in\{0,1\}^{n}} f_{1}(x)=x^{T} A_{1} x$

$\min _{x \in\{0,1\}^{n}} f_{2}(x)=x^{T} A_{2} x$ 
$\min _{x \in\{0,1\}^{n}} f_{\mathrm{r}}(x)=x^{T} A_{r} x$

S.t. $B x \geq b, x^{T} C_{1} x \geq \alpha_{1}, x^{T} C_{2} x \geq \alpha_{2}, \ldots, x^{T} C_{k} x \geq \alpha_{k}, x \in\{0,1\}^{n}, \quad \alpha_{i}$ are positive constants.

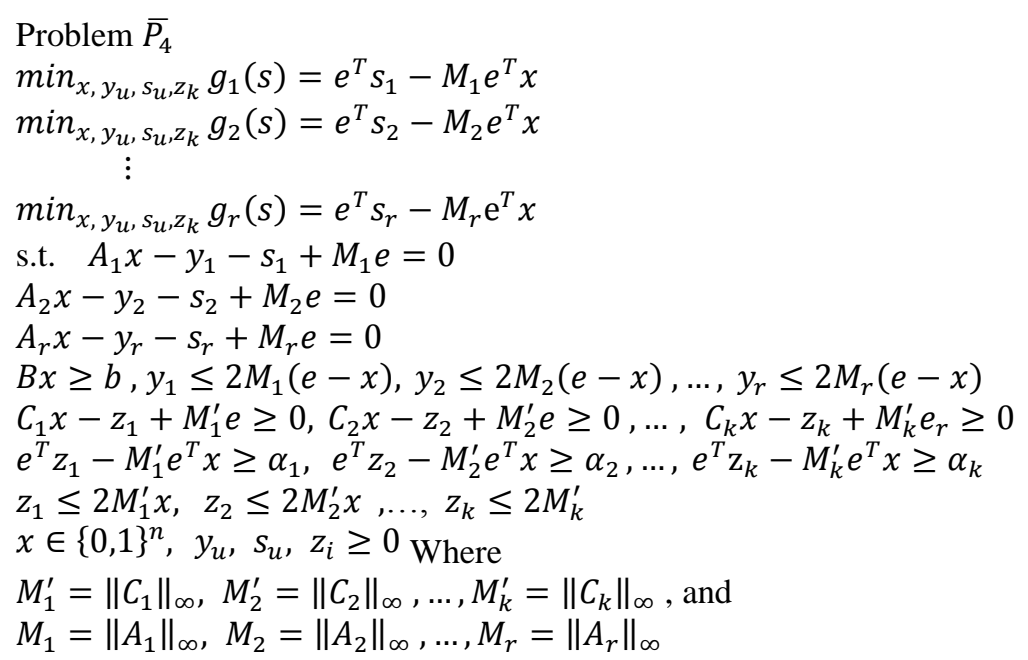

Theorem 4.2: $P_{4}$ has a Pareto optimal solution $x^{0}$ if there existed $y_{u}{ }^{0}, s_{u}{ }^{0}, z_{i}{ }^{0}$ such that $\left(x^{0}, y_{u}{ }^{0}, s_{u}{ }^{0}, z_{i}{ }^{0}\right)$ is a Pareto optimal solution of $\overline{P_{4}}$.

Proof: Necessity. The proof is similar to the proof of Theorem 2.2.

From the proof of the theorem 4.1, it is obvious that we only need to show that if $x^{0}$ is a Pareto optimal solution of the problem $P_{4}$ then there exists vectors $z_{i}{ }^{0}$ such that every component of each vector is non-negative and the following constraints are satisfied:

$C_{1} x-z_{1}+M_{1}^{\prime} e \geq 0, C_{2} x-z_{2}+M_{2}^{\prime} e \geq 0, \ldots, C_{r} x-z+M_{2}^{\prime} e_{r} \geq 0$

$e^{T} z_{1}-M_{1}^{\prime} e^{T} x \geq \alpha_{1}, e^{T} z_{2}-M_{2}^{\prime} e^{T} x \geq \alpha_{2}, \ldots, e^{T} z_{k}-M_{k}^{\prime} e^{T} x \geq \alpha_{k}$

$z_{1} \leq 2 M_{1}^{\prime} x, z_{2} \leq 2 M_{2}^{\prime} x, \ldots, z_{k} \leq 2 M_{k}^{\prime} x$

$C_{1} x^{0}-z_{1}+M_{1}^{\prime} e \geq 0, C_{2} x^{0}-z_{2}+M_{2}^{\prime} e \geq 0, \ldots, C_{r} x^{0}-z_{r}+M_{r}^{\prime} e \geq 0$

$e^{T} z_{1}{ }^{0}-M_{1}^{\prime} e^{T} x \geq \alpha_{1}, e^{T} z_{2}{ }^{0}-M_{2}^{\prime} e^{T} x \geq \alpha_{2}, \ldots, e^{T} z_{k}{ }^{0}-M_{2}^{\prime} e^{T} x \geq \alpha_{k}$

$z_{1}{ }^{0} \leq 2 M_{1}^{\prime} x^{0}, z_{2}{ }^{0} \leq 2 M_{2}^{\prime} x^{0}, \ldots, z_{k}{ }^{0} \leq 2 M_{k}^{\prime} x^{0}$

From (20), note that if $x_{i}{ }^{0}=0$ then $z_{i}{ }^{0}=0$.

Similar to the proof of Theorem.2.1,

$e^{T} z_{1}{ }^{0} \geq\left(x^{0}\right)^{T} z_{1}{ }^{0}, \quad e^{T} z_{2}{ }^{0} \geq\left(x^{0}\right)^{T} z_{2}{ }^{0}, \ldots, e^{T} z_{k}{ }^{0} \geq\left(x^{0}\right)^{T} z_{k}{ }^{0}$

Since component of $z_{i}^{0}$ are real numbers and

$C_{1} x^{0}+M_{1}^{\prime} e \geq 0, C_{2} x^{0}+M_{2}^{\prime} \mathrm{e} \geq 0, \ldots, \mathrm{C}_{\mathrm{r}} \mathrm{x}^{0}+\mathrm{M}_{\mathrm{r}}^{\prime} \mathrm{e} \geq 0$

For every $\mathrm{i}$, where $\mathrm{x}_{\mathrm{i}}{ }^{0}=1$, we can choose $\mathrm{z}_{\mathrm{i}}{ }^{0}>0$ such that $\left(\mathrm{C}_{1} \mathrm{x}^{0}+\mathrm{M}_{1}^{\prime} \mathrm{e}\right)=\mathrm{z}_{\mathrm{i}}{ }^{0}$. Therefore (18) and (20) are satisfied.

Multiplying (18) by $\left(\mathrm{x}^{0}\right)^{\mathrm{T}}$, from (21) we obtain that

$\left(\mathrm{x}^{0}\right)^{\mathrm{T}} \mathrm{C}_{1} \mathrm{x}^{0}+\mathrm{M}_{1}^{\prime} \mathrm{ex}^{0}=\left(\mathrm{x}^{0}\right)^{\mathrm{T}} \mathrm{z}_{1}{ }^{0}$

$\left(\mathrm{x}^{0}\right)^{\mathrm{T}} \mathrm{C}_{2} \mathrm{x}^{0}+\mathrm{M}_{2}^{\prime} \mathrm{ex}^{0}=\left(\mathrm{x}^{0}\right)^{\mathrm{T}} \mathrm{z}_{2}{ }^{0}$

$\left(\mathrm{x}^{0}\right)^{\mathrm{T}} \mathrm{C}_{\mathrm{k}} \mathrm{x}^{0}+\mathrm{M}_{\mathrm{k}}^{\prime} \mathrm{e} \mathrm{x}^{0}=\left(\mathrm{x}^{0}\right)^{\mathrm{T}} \mathrm{z}_{\mathrm{k}}{ }^{0}$

And as $\mathrm{x}^{0}$ is an optimal solution of the problem $\mathrm{P}_{4}$ then (19) is satisfied:

$\mathrm{e}^{\mathrm{T}} \mathrm{z}_{1}{ }^{0}-\mathrm{M}_{1}^{\prime} \mathrm{e}^{\mathrm{T}} \mathrm{x}^{0}=\left(\mathrm{x}^{0}\right)^{\mathrm{T}} \mathrm{C}_{1} \mathrm{x}^{0} \geq \alpha_{1}$

$\mathrm{e}^{\mathrm{T}} \mathrm{z}_{2}{ }^{0}-\mathrm{M}_{2}^{\prime} \mathrm{e}^{\mathrm{T}} \mathrm{x}^{0}=\left(\mathrm{x}^{0}\right)^{\mathrm{T}} \mathrm{C}_{2} \mathrm{x}^{0} \geq \alpha_{2}$

$\mathrm{e}^{\mathrm{T}} \mathrm{z}_{\mathrm{k}}{ }^{0}-\mathrm{M}_{\mathrm{k}}^{\prime} \mathrm{e}^{\mathrm{T}} \mathrm{x}^{0}=\left(\mathrm{x}^{0}\right)^{\mathrm{T}} \mathrm{C}_{\mathrm{k}} \mathrm{x}^{0} \geq \alpha_{\mathrm{k}}$

Sufficiency: The proof is similar.

The number of new additional continuous variables needed for the reduction is $O(n)$. In the case of $k$ quadratic constraints the number of new variables is $\mathrm{O}(\mathrm{kn})$. The number of additional linear constraints is also $\mathbf{O}(\mathbf{k n})$.

\section{Conclusion}

A linearization technique is developed to reduce a multi-objective multi-quadratic 0-1 programming problem to multiobjective linear mixed 0-1 programming problems. Here multi-level and multi-objective programming problem is used. It is observed that the number of new additional continuous variables needed for the reduction is $\mathrm{O}(\mathrm{n})$. In the case of $\mathrm{k}$ quadratic constraints the number of new variables is $\mathrm{O}(\mathrm{kn})$. The number of additional linear constraints is also $\mathrm{O}(\mathrm{kn})$. 


\section{References}

[1] C.A. Floudas and V. Visweswaran, Quadratic optimization, in: R. Horst, P.M. Paradalos (Eds.), Handbook of Global Optimization, Kluwer Academic Publishers, Dordrecht, 1995, 217-269.

[2] C.T. Chang and C.C. Chang, A linearization method for mixed 0-1 polynomial programs, Computers and Operations Research 27 (2000) 1005-1016.

[3] F. Glover and E. Woolsey, Further reduction of 0-1 polynomial programs to 0-1 linear programming, Operations Research, 21 (1) (1973) 156161.

[4] F. Glover and E. Woolsey, Converting the 0-1 polynomial programming program, Operations Research, 22(1) (1974) 180-182.

[5] L. J. Watters, Reduction of integer polynomial programming to 0-1 linear programming problems, Operations Research 15 (1967) $1171-1174$.

[6] P.M. Pardalos, L.D. Iasemidis, D.S. Shiau et al, Quadratic binary programming and dynamic system approach to determine the predictability of epileptic seizures, Journal of Combinatorial Optimization 5 (1) (2001) 9-26.

[7] P.M. Pardalos, L.D. Iasemidis, D.S. Shiau et al, Prediction of human epileptic seizures based on optimization and phase changes of brain electrical activity, Optimization Methods and Software 18 (1) (2003) 81-104.

[8] P.M. Pardalos, W. Chaovalitwongse, O.A. Prokopyev, A new linearization technique for multi-quadratic 0-1 programming problems, Operations Research Letters 32 (2004) 517-522.

[9] P.M. Pardalos, W. Chaovalitwongse, L.D. Iasemidis et al, Seizure warning algorithm based on optimization and nonlinear dynamics, Mathematical Programming Ser. B 101 (2004) 365-385.

[10] R. Kapoor and S.R. Arora, Linearization of 0-1 multi-quadratic fractional programming problem, Asia Pacific Journal of Operational Research 26(1) (2009) 59-84.

[11] S. Acharya and M.P. Biswal, Linearization technique for multi-choice quadratic Programming Problem, International Journal of Optimization, Theory, Methods and Applications, 3(1) (2011) 45-62.

[12] W.P. Adams and H.D. Sherali, Linearization strategies for a class of 0-1 mixed integer programming problems, Operations Research 38 (2) (1990) 217-226.

[13] X. He, A. Chen and W. Chaovalitwongse et al, An improved linearization technique for a class of quadratic 0-1 programming problems, Optimization Letters 6(1) (2012) 31-41. 